\title{
Comparison of Multi-Detector Computed Tomography Coronary Angiography with Invasive Coronary Angiography in Patients with Coronary Artery Disease
}

\section{Koroner Arter Hastalığı Olan Hastalarda Çok Dedektörlü Bilgisayarlı Tomografi Koroner Anjiografi ile İnvaziv Koroner Anjiografinin Karşılaştırılması}

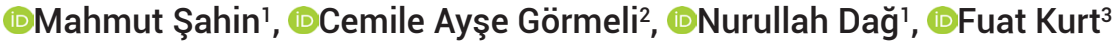 \\ 'Malatya Training and Research Hospital, Department of Radiology, Malatya, Turkey \\ ${ }^{2}$ Halic University, School of Medicine, Department of Radiology, Istanbul, Turkey \\ ${ }^{3}$ Malatya Training and Research Hospital, Department of Cardiology, Malatya, Turkey \\ Copyright@Author(s) - Available online at www.dergipark.org.tr/tr/pub/medr \\ Content of this journal is licensed under a Creative Commons Attribution-NonCommercial 4.0 International License.
}

\begin{abstract}
Aim: This study aimed to investigate the accuracy of multi-detector computed tomography (MDCT) coronary angiography via comparing with the invasive coronary angiography (ICA).

Material and Method: Sixty-three patients (42 male, 21 female) that presented with chest pain and underwent MDCT, followed by ICA within one month were evaluated. The age of the patients ranged from 35 to 75 years. The coronary arteries were examined over a total of 15 segments according to American Heart Association classification. The detected stenoses of coronary artery were divided into four groups; non-obstructive (1-49\%), significant stenosis (50-74\%), high-grade stenosis (75-99\%), and occlusion (100\%). Segment-based and patient-based analyses were performed. The results of MDCT coronary angiography and ICA were compared. The sensitivity, specificity, positive predictive and negative predictive values were calculated by comparing the MDCT coronary angiography and ICA data.

Results: In the segment-based analysis, regardless of the stenosis rate, the sensitivity was $90.8 \%$, specificity was $95 \%$, positive predictive value was $82.7 \%$ and negative predictive value was $97.5 \%$. In the segment-based analysis regarding the detection of $\geq 50 \%$ stenosis, the sensitivity specificity, positive predictive and negative predictive values were $89.6 \%, 95.9 \%, 59.0 \%$, and $99.2 \%$, respectively. In the patient-based analysis regarding the detection of $\geq 50 \%$ stenosis, the sensitivity was $96.2 \%$, specificity was $66.6 \%$, positive predictive value was $68.4 \%$, and negative predictive value was $96 \%$.

Conclusion: MDCT coronary angiography is an examination that can be used as a non-invasive method for patients in the low and medium risk group for coronary artery disease.
\end{abstract}

Keywords: Multi-detector computed tomography; invasive coronary angiography; coronary artery disease

Öz

Amaç: Bu çalışma, çok dedektörlü bilgisayarlı tomografı (ÇDBT) koroner anjiyografınin doğruluğunu, invazif koroner anjiyografı (iKA) ile karşılaştırarak incelemeyi amaçladı.

Materyal ve Metot: Göğüs ağrısı ile başvurup ÇDBT koroner anjiografı çekilen ve bunu takiben bir ay içerisinde IKA yapılan hastalar çalışmaya alındı. Çalışma grubumuzda yaşları 35-75 arasında değişen 42'si erkek, 21'i kadın toplam 63 hasta vardı. Koroner arterler American Heart Association sınıflamasına göre toplam 15 segment üzerinden incelendi. Tespit edilen koroner arter stenozları 4 gruba ayrıldı; non-obstrüktif (\%1-49), anlamlı stenoz (\%50-74), yüksek dereceli stenoz (\%75-99) ve oklüzyon (\%100). ÇDBT sonuçları IKA ile karşılaştırılarak segment bazlı ve hasta bazlı analizler yapıldı. Elde ettiğimiz veriler İKA sonuçları ile kıyaslanarak sensivite, spesifıte, pozitif prediktif değer ve negatif prediktif değerleri hesaplandı.

Bulgular. Çalışmamızda segment bazlı analizde stenoz oranlarına bakılmaksızın yapılan değerlendirmede sensitivite \%91.5, spesifıte $\% 95$, pozitif prediktif değer \%84.7 ve negatif prediktif değer \%97.4, segment bazlı $\geq \% 50$ stenozları saptamada sensitivite \%89.6, spesifıte \%95.9, pozitif prediktif değer \%59.0 ve negatif prediktif değer \%99.2, hasta bazlı değerlendirmelerimizde $\geq \% 50$ stenoz tespit etmede sensitivite $\% 96.2$, spesifite $\% 66.6$, pozitif prediktif değer \%68.4 ve negatif prediktif değeri \%96 bulduk.

Sonuç: ÇDBT koroner anjiografi, koroner arter hastalığı açısından düşük ve orta risk grubunda yer alan hastalarda non-invaziv bir yöntem olarak kullanılabilir bir tetkiktir.

Anahtar Kelimeler : Multi-dedektör bilgisayarlı tomografi; invaziv koroner anjiografı; koroner arter hastalığı 


\section{INTRODUCTION}

Cardiovascular diseases are one of the leading causes of death worldwide (1). The most common cardiovascular disease is coronary artery disease (CAD), which has the greatestmortalityandmorbidityrates (2). Invasivecoronary angiography (ICA) is accepted as the gold standard in the diagnosis of CAD. It has significant advantages, such as having high spatial and temporal resolution and facilitating the use of additional interventional methods for treatment. However, ICA has an invasive nature and is costly, causes certain complications, and is insufficient in characterizing plaques. For these reasons, a non-invasive, more cost-effective imaging method with high diagnostic sensitivity was needed (3).

In the last years, multi-detector computed tomography (MDCT) coronary angiography has been increasingly used for the evaluation of CAD. MDCT coronary angiography is a non-invasive imaging method that has a high spatial and temporal resolution, can show anatomical details from multiple perspectives. MDCT coronary angiography can evaluate other anatomical structures of the heart as well as coronary arteries. In addition, it can distinguish the coronary artery wall-atherosclerotic plaque border and characterize plaques by providing three-dimensional and cross-sectional images rather than projection images (4). MDCT coronary angiography provides independent prognostic information on mortality and estimated cardiac events in patients with known or suspected CAD. The use of firstly MDCT coronary angiography is recommended for patients with a low or moderate risk of CAD (5).

In this study, we aim to evaluate the diagnostic accuracy of MDCT coronary angiography regarding ICA in the diagnosis of CAD.

\section{MATERIAL AND METHOD}

\section{Patient Selection and Preparation}

The retrospective study was approved by the Inonu University Medical Faculty Ethical Committee. (2015/16). 138 patients who underwent MDCT coronary angiography in the radiology department between February 2015 and January 2016 were included in the study. In the patients included in the study, ICA was performed within 1 month in the cardiology clinic after MDCT coronary angiography. Patients with contrast material allergy, renal failure, advanced heart failure, hyperthyroidism, epilepsy, respiratory distress, Reynaud's syndrome, atrioventricular block, were excluded from the study. In addition, forty-six patients with a history of bypass surgery and / or coronary artery stenting, 18 patients with a duration of more than 1 month between MDCT coronary angiography and ICA, and 11 patients with insufficient MDCT images due to motion, cardiac arrhythmia or respiratory artefacts were excluded from the study.

\section{MDCT Protocol}

The heart rate of pre-scan patients was aimed to be 70 beats / min. Beta-blocker therapy was administered to patients with a high heart rate under the supervision of a cardiologist. In all cases, before MDCT coronary angiography, unenhanced CT was performed to determine the calcium load (calcium score) of the coronary arteries. Calcium scoring was automatically calculated by software specifically designed to mark calcified areas. MDCT coronary angiography was performed with 64-slice (Aquillon; Toshiba Medical Systems) and 256-slice (Somatom Definition Flash; Siemens Healthcare) CT devices. The duration of the examination in the 64-slice MDCT device varied between the cases with the average duration being calculated as 7-10 sec. Imaging was undertaken in the routine spiral mode applying standard protocols. In the 256-slice CT device, three different imaging protocols were used according to the heart rate of the patients. The duration of imaging was between 5 and $8 \mathrm{sec}$. For patients with a heart rate of 60 beats / min or less, the Flash Spiral scan protocol was applied. In this mode, CT angiography images were obtained at a single heart rate with an extremely low dose of radiation. In patients without arrhythmia but having a heart rate of $60-90$ beats / min., the adaptive prospective electrocardiogram (ECG)-triggered mode was used. The patients with arrhythmia or a heart rate of $\geq 90$ were scanned using the routine spiral mode.

Before the MDCT procedure, scanogram images covering the carina and the base of the heart were used to determine the area to be scanned. Then, ECG-recorded helical images of the whole heart from the carina to the base were obtained during inspiration. A contrast agent $(75-95 \mathrm{ml})$ with high iodine concentration $(\geq 350 \mathrm{mg} /$ $\mathrm{mL}$ ) was administered using an autoinjector at a rate of $5 \mathrm{ml} / \mathrm{sec}$ for 64 -slice scans and $6 \mathrm{ml} / \mathrm{sec}$ for 256 -slice scans. Following the contrast enhancement, $50 \mathrm{~mL}$ saline bolus was injected at a rate of $5 \mathrm{~mL} / \mathrm{sec}$ to reduce artefacts in the right heart and allow the examination of contrast material in dead spaces (line, antecubital vein and right heart). An automated dual-syringe injector (CT motion, Ulrich medical) was used for the administration of contrast medium and saline.

\section{Invasive Coronary Angiography}

Conventional coronary angiography was performed with the transfemoral Judkins approach, and the right coronary artery (RCA) and left main coronary artery branches were displayed in different projections. Stenosis rates were compared on two different planes with normal segments on the proximal side and recorded by a cardiologist that did not know the findings of MDCT coronary angiography.

\section{Evaluation and Interpretation of Images}

During imaging, the heart rate and ECG tracing were recording retrospectively for the spiral mode and prospectively for the adaptive mode. The images were then transferred to workstations for analysis (Syngo Via; Siemens Medical Solutions, Vitrea; Toshiba Medical Systems). Thin-axial data was used to obtain images in the formats of two-dimensional maximum intensity 
projection (MIP), multiplanar reconstruction (MPR), and three-dimensional volumetric display. The MPR and MIP images were used to evaluate arterial lumens, arterial wall and heart chambers, and three-dimensional images were utilized to assess coronary artery anatomy and stenosis. The volumetric rendering technique was adopted to demonstrate the complicated anatomic threedimensional characteristics of coronary arteries and to obtain details that might have been overlooked in the examination of images on the axial plane.

In the 64-slice CT procedure, reconstruction percentages corresponding to $35-40 \%$ in ECG tracing and $70 \%$ in left anterior descending (LAD) and left circumflex artery (LCX) coronary arteries were used for RCA. For the 256-slice CT images, the best systolic and diastolic reconstructions were automatically detected on the workstation. Thus, coronary arteries were examined in detail on images with minimal artefacts. All images were evaluated by a radiologist experienced in cardiovascular radiology. The coronary arteries were examined over 15 segments according to the American Heart Association (AHA) classification. On this basis, RCA consisted of segments $1-4$, the left main coronary artery segment 5 , LAD segments 6-10, and LCX segments 11-15.

Taking the stenosis-free luminal diameter proximal to the stenosis arterial segment as a reference, $50 \% \geq$ narrowing of the luminal diameter in the stenosis segment was interpreted as obstructive CAD. The detected coronary artery stenoses were divided into four groups; normal, non-obstructive (1-49\%), significant stenosis (50-74\%), high-grade stenosis (75-99\%), and occlusion (100\%).

The data obtained were compared with the results of ICA, and the sensitivity, specificity, and positive and negative predictive values were calculated. Segment- and patientbased analyses were performed on the data. In the segment-based analysis, the sensitivity, specificity, and positive and negative predictive values were calculated for the detection of lesions in all segments regardless of stenosis rate and for identification of those that caused $\geq 50 \%$ and $\geq 75 \%$ stenosis. In the patient-based analysis, these values were calculated only for the determination of lesions that caused $\geq 50 \%$ and $\geq 75 \%$ stenosis. In the same analysis, the detection of stenosis by ICA and MDCT at the same segment level in a coronary artery was evaluated as an indication of a true positive case.

According to their morphology, plaques were characterized as calcified, soft or mixed. A plaque was considered to be soft if calcification was not observed or was minimal $(0-130 \mathrm{HU})$, mixed if it was calcified but had soft components (> $130 \mathrm{HU})$, and calcified if the whole plaque was calcified (> $130 \mathrm{HU}$ ). ICA and MDCT images of 2 patients are shown in figures 1 and 2 .

The statistical analysis was performed using the Statistical Package for the Social Sciences (SPSS) version 17.0. Categorical variables were presented as counts and percentages. Sensitivity, specificity, positive predictive value and negative predictive value for MDCT to detect significant stenosis were calculated from the chi-square test of contingency.

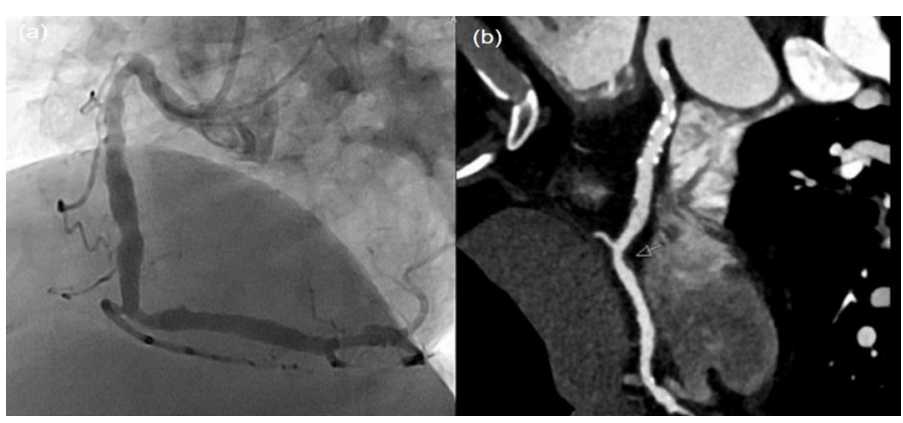

Figure 1. An ICA image (a) and MDCT curved MPR reconstruction image (b) of a 65-year-old male patient with a soft plaque that caused approximately $50 \%$ narrowing of the lumen following the RCA acute marginal branch. In addition, in the ICA image, at the proximal RCA level, calcified plaques causing luminal irregularity but no significant stenosis are observed.

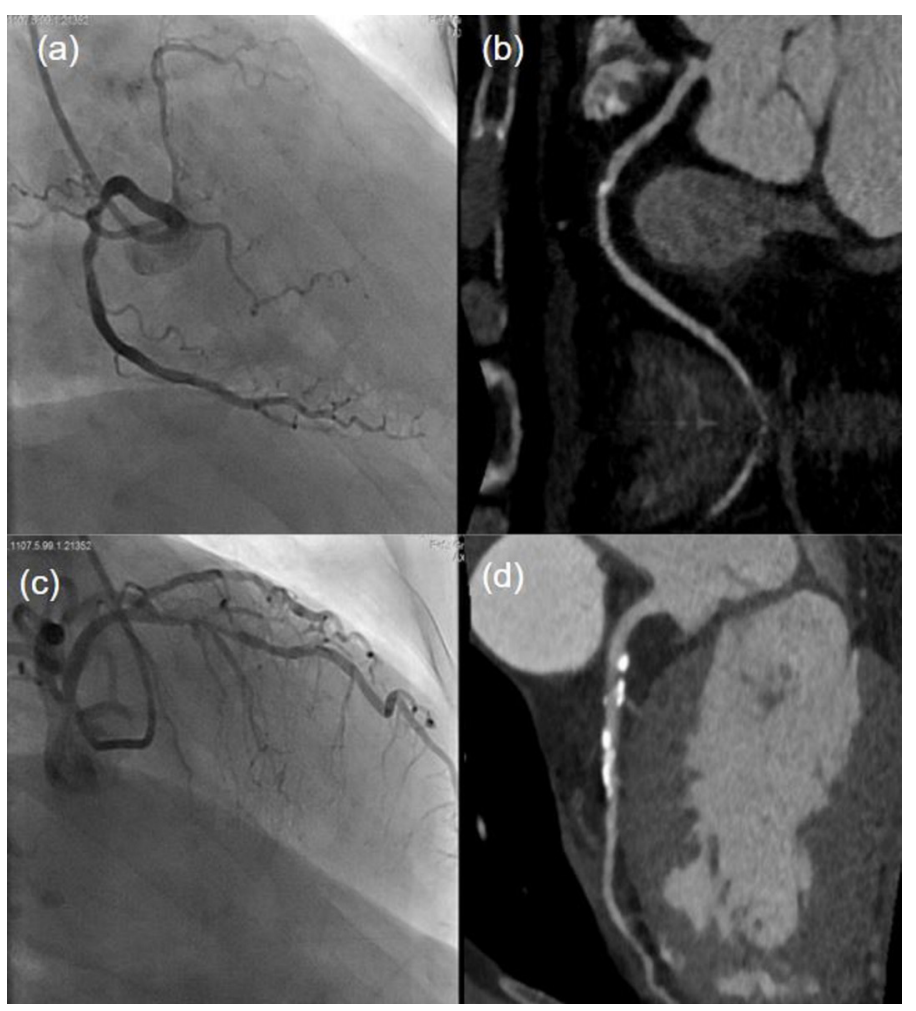

Figure 1. An ICA image (a) and MDCT curved MPR reconstruction image (b) of a 74-year-old female patient with a discrete calcified plaque that caused $50-74 \%$ narrowing of the lumen before the RCA acute marginal branch. In the same patient, the ICA (c) and curved MPR (d) images show a tubulary calcified plaque following LAD D1 causing $50-74 \%$ stenosis. Another calcific plaque is observed in the LAD proximal segment without significant stenosis.

\section{RESULT}

The sample of the study consisted of 63 patients, 42 male (42.6\%) and 21 female (33.3\%), aged 35 to 75 years. A total of 946 coronary arterial segments were evaluated by ICA, and stenosis was detected in 196 of these segments at various levels and rates. Of the segments with stenosis, 
$90.8 \%$ ( $n=178$, true positive result) were also detected by MDCT coronary angiography.

Using MDCT, stenosis was detected in 215 segments at various levels, of which 37 were evaluated as normal in ICA (false-positive result). A total of 712 segments were evaluated as normal (true-negative result) using MDCT coronary angiography and ICA. Eighteen segments were reported to be normal in MDCT coronary angiography but had stenosis according to ICA (false-negative result).

According to the findings of MDCT coronary angiography and ICA, obstructive CAD was not present in 851 of 945 segments. Six segments were evaluated as nonobstructive CAD or normal by MDCT coronary angiography but defined as obstructive CAD according to ICA. Both ICA and MDCT angiography revealed obstructive CAD in 52 segments. Thirty-six segments were identified as obstructive CAD by MDCT coronary angiography, but defined as non-obstructive CAD or normal by ICA.

Concerning the detection of segments with high-grade stenosis (75-99\%) and occlusion (100\%), we obtained the following results: Of the 945 segments, 911 had no high-grade stenosis or occlusion according to both MDCT coronary angiography and ICA; nine segments were evaluated as normal or having stenosis below $75 \%$ by MDCT coronary angiography but identified as high-grade stenosis or occlusion by ICA; 16 segments had high-grade stenosis or occlusion according to both imaging techniques; and nine segments were interpreted to have high-grade stenosis or occlusion by MDCT coronary angiography but evaluated as having less than $75 \%$ stenosis or being normal in ICA. Segment-based assessment results were summarized in tables 1 and 2 .
In the patient-based assessment, the presence of stenosis above the specified threshold in any segment of coronary arteries revealed by both MDCT coronary angiography and ICA was interpreted as a true-positive case. Here, we assumed a threshold of $\geq 50 \%$ for obstructive CAD and $\geq 75 \%$ for severe stenosis. In the analysis of 63 patients, 27 patients were found to have $\geq 50 \%$ stenosis according to ICA, and 26 of these patients were also identified by MDCT coronary angiography (true-positive patient). There was only one patient (false-negative) who was evaluated as having $\geq 50 \%$ stenosis in ICA, but was not identified by MSCT coronary angiography. In the obstructive CAD analysis, 24 patients were evaluated as normal by both MDCT coronary angiography and ICA (true-negative patients). Twelve patients had $\geq 50 \%$ stenosis according to MDCT coronary angiography, but were evaluated as normal or having non-obstructive CAD by ICA (falsepositive patients).

In the analysis of high-grade stenosis ( $\geq 75 \%), 16$ patients were identified to have high-grade stenosis by ICA, of whom only 11 were also evaluated the same in MDCT coronary angiography. For the remaining five patients, MDCT coronary angiography revealed $50-74 \%$ stenosis in three patients and $1-49 \%$ stenosis in two patients. Forty-four patients were evaluated as having normal findings by both MDCT coronary angiography and ICA. Lastly, three patients that were found to have high-grade stenosis in MDCT coronary angiography were assessed as having normal findings or less severe stenosis in ICA. Concerning the characterization of plaques identified by MDCT angiography, $48.3 \%(n=117)$ were mixed, $39.2 \%(n$ $=95)$ were calcified, and $12.3 \%(n=30)$ were soft. Patient-

Table 1. The true-positive, true-negative, false-positive, and false-negative results of MDCT angiography for the detection of stenosis in all segments (segment-based assessment)

ICA

STENOSIS PRESENT

PRESENT

ABSENT

PRESENT

ABSENT

PRESENT
178

18

52

6

16

9
STENOSIS ABSENT

37

712

36

851

9

911

Table 2. The sensitivity, specificity, and positive and negative predictive values of MDCT angiography for the detection of stenosis (segment-based assessment)

$\begin{array}{lcccc} & \text { Sensitivity } & \text { Specificity } & \text { Positive predictive value } & \text { Negative predictive value } \\ \text { STENOSIS REGARDLESS OF RATE } & 90.8 \% & 95 \% & 82.7 \% & 97.5 \% \\ \geq \text { 50\% STENOSIS } & 89.6 \% & 95.9 \% & 59 \% & 99.2 \% \\ \geq 75 \% \text { STENOSIS } & 64 \% & 99 \% & 64 \% & 99 \%\end{array}$


Table 3 . The true positive, true negative, false positive and false negative results of MDCT in detecting $\geq 50 \%$ and $\geq 75 \%$ stenosis in all patients (patient-based assessment)

\begin{tabular}{|c|c|c|c|c|}
\hline & & & ICA & \\
\hline & & & STENOSIS PRESENT & STENOSIS \\
\hline & $\geq 50 \%$ STENOSIS & & ABSENT & $99.2 \%$ \\
\hline MDCT & & PRESENT & 26 & 12 \\
\hline IVI & & ABSENT & 1 & 24 \\
\hline & $\geq 75 \%$ STENOSIS & PRESENT & 11 & 3 \\
\hline & & ABSENT & 5 & 44 \\
\hline & Sensitivity & Specificity & Positive Predictive Value & Neqative Predictive Value \\
\hline$\geq 50 \%$ STENOSIS & 96.2 & 66.6 & 68.4 & 96 \\
\hline$\geq 75 \%$ STENOSIS & 68.7 & 93.6 & 78.5 & 89 \\
\hline
\end{tabular}

based assessment results were summarized in tables 3 and 4.

\section{DISCUSSION}

In our study, we found that MDCT coronary angiography provided high sensitivity and negative predictive values in the segment and patient-based analyzes.

ICA is the gold standard method in the diagnosis of CAD. In the presence of CAD, ICA shows the luminal diameter, stenosis rate, and luminal irregularity with high resolution $(3,6)$. An important advantage of ICA is that it facilitates the use of interventional procedures, such as balloon or stent placement in the stenotic region under emergency or elective conditions according to the clinical findings from the examination of the patient (6). However, since ICA indirectly displays the lumen of the coronary arteries secondary to contrast enhancement, it only provides information about the lumen and does not allow direct observation of the arterial wall. For this reason, ICA does not offer any insight into the character of an atherosclerotic plaque or its rupture tendency (7). Lumen stenosis is usually determined by the proportion of a stenotic segment to the normal segment proximal to the stenosis. In the presence of diffuse atherosclerotic CAD, if there is no normal arterial segment, ICA may underestimate the stenosis rate $(6,7)$. In addition, outward displacement of plaques (positive remodelling) may cause normal visualization of the luminal diameter despite the presence of significant CAD. Furthermore, the invasive nature of ICA brings certain risks, the severity of which is related to the skill and experience of the angiographer, the stability of the patient's clinical symptoms, and the diffuseness of CAD. The most important complications are stroke, myocardial infarction, and death (6-8). The high cost of ICA and the requirement of interventional procedures only in one third of cases are among the other disadvantages of this procedure. Therefore, a more cost-effective and non-invasive imaging method is necessary for diagnostic purposes $(3,6,7)$.

MDCT coronary angiography has been used since 1998 to visualize vascular structures outside the coronary artery system with the advances in technology making it possible to visualize long-range and high-resolution images. Since the heart is a moving organ, MDCT started to be used for imaging coronary arteries only after the ECG tracing technology was introduced to MDCT devices (8). Involving the use of synchronous imaging with ECG and reconstruction methods, having a high spatial and temporal resolution, and being able to perform faster volume scanning, MDCT allows detecting coronary artery stenosis with high sensitivity at low pulse following appropriate preparations (8). Furthermore, features such as increased number of detectors, reduced gantry rotation period, decreased cross-sectional thickness, and increased X-ray utilization factor in MDCT devices have increased the use of MDCT coronary angiography in CAD imaging (7-9).

The advances in CT technology have eliminated the problems with temporal resolution, which is a critical parameter in the visualization of the heart, a moving organ. High diagnostic values have been achieved using 64-slice MDCT, and this method has been accepted as a non-invasive alternative to ICA in patients suspected of having coronary artery stenosis (10).

In a meta-analysis of 29 studies involving 2,024 patients and the comparison of MDCT with ICA, the sensitivity, specificity, and positive and negative predictive values of MDCT in detecting lesion were reported to be $81 \%, 93 \%$, $67.8 \%$, and $96.5 \%$, respectively, regardless of the stenosis rate (11). Similarly, in the current study, we found $90.8 \%$ sensitivity, $95 \%$ specificity, $82.7 \%$ positive predictive value, and $97.5 \%$ negative predictive value.

Ehara et al. conducted a coronary angiography study using a 64-slice CT device and evaluated 884 segments in 69 patients, and reported that the sensitivity, specificity, 
positive predictive value, and negative predictive value for $\geq 50 \%$ stenosis detection were $90 \%, 94 \%, 89 \%$, and $95 \%$, respectively (12). In another study, Hans et al. compared the findings obtained by 64 -slice CT with ICA in 50 patients, and found that for $\geq 50 \%$ stenosis, the sensitivity, specificity, and positive and negative predictive values of MDCT were $81.8 \%, 97.7 \%, 88.9 \%$, and $95 \%$, respectively (13). In the segment-based analyses of the current study, when we compared the MDCA results with those of ICA in detecting $\geq 50 \%$ stenosis, we found $89.6 \%$ sensitivity, $95.9 \%$ specificity, $59.0 \%$ positive predictive value, and $99.2 \%$ negative predictive value. These results are similar to those reported by most of the MDCT coronary angiography studies conducted in recent years. Our positive predictive value was low because some of the segments evaluated as $50-74 \%$ stenosis in MDCT coronary angiography were interpreted as $1-49 \%$ stenosis by ICA. In another study conducted with 104 patients using a 256-slice MDCT device, segment-based analyses revealed $93.5 \%$ sensitivity, $95 \%$ specificity, $77.6 \%$ positive predictive value, and $98.7 \%$ negative predictive value for the detection of $\geq 50 \%$ stenosis (14). In our study, although we utilized both 64-slice and 256-slice MDCT devices, the former was used for the imaging of most patients. When we compare our results with the above-mentioned study that only used a 256-slice MDCT device, there are no significant differences. Similar to our results, their positive predictive value was also low. Today, MDCT coronary angiography is indicated for use in obstructive CAD in patients with low to moderate risk. This is supported by large scientific communities due to the high negative predictive value of MDCT angiography for obstructive CAD (5). In a multi-center study of 291 patients, although the negative predictive value was high, the positive predictive value was found to be low (15).

Alexander et al. evaluated 798 segments in 64 patients using 64-slice MDCT. According to their segment-based analysis, compared to ICA, the sensitivity and specificity of MDCT for the detection of $\geq 75 \%$ stenosis were $80 \%$ and $97 \%$, respectively (16). Our segment-based analysis revealed $64 \%$ sensitivity, $99 \%$ specificity, $64 \%$ positive predictive value, and $99 \%$ negative predictive value for the detection of $\geq 75 \%$ stenosis. We consider that our lower sensitivity value is due to misinterpretations regarding stenosis rates. The segments that we evaluated as $50-$ $74 \%$ stenosis in MDCT but defined as $\geq 75 \%$ stenosis in ICA reduced the sensitivity value. Therefore, despite the high sensitivity percentages for lesion detection, we obtained lower sensitivity in detecting stenosis rates. Similarly, the lower positive predictive value may be explained by the interpretation of some segments as $\geq$ $75 \%$ stenosis in MDCT coronary angiography but as $50-$ $74 \%$ stenosis in ICA.

In a prospective and multi-center study of 230 consecutive patients presenting with chest pain but no previously known CAD, Budoff et al. investigated the diagnostic accuracy of 64-slice MDCT coronary angiography based on $50 \%$ and $70 \%$ threshold values (17). Their patient- based analysis on the data revealed $95 \%$ sensitivity, $83 \%$ specificity, $64 \%$ positive predictive value, and $99 \%$ negative predictive value for the detection of $\geq 50 \%$ stenosis. Similarly, for the detection of $\geq 70 \%$ stenosis, the sensitivity, specificity, and positive and negative predictive values were reported as $94 \%, 83 \%, 48 \%$, and $99 \%$, respectively. In another study conducted with 104 patients using 256-slice MDCT, the patient-based analysis showed $98.8 \%$ sensitivity, $50 \%$ specificity, $92.4 \%$ positive predictive value, and $87.5 \%$ negative predictive value for the detection of $\geq 50 \%$ stenosis (14). In our patientbased analysis, compared to ICA, we found the sensitivity, specificity, and positive and negative predictive values of MDCT to be $96.2 \%, 66.6 \%, 68.4 \%$, and $96 \%$, respectively for $\geq 50 \%$ stenosis detection and $68.7 \%, 93.6 \%, 78.5 \%$, and $89 \%$, respectively for $\geq 75 \%$ stenosis detection. We consider that the low positive predictive values for the $\geq 50 \%$ and $\geq 75 \%$ thresholds and the low sensitivity for the $\geq 75 \%$ threshold are not related to the detection of lesions, but are due to inaccuracies in determining the rates of stenosis they cause in the lumen.

Many studies investigating the accuracy of MDCT coronary angiography have reported high false-positive results and low positive predictive values. Among the factors that increase false positives are technical problems and motion artefacts that degrade image quality. Common high-density calcified plaques in coronary arteries not only make it difficult to evaluate MDCT coronary angiography but also limit the validity of the results. The presence of intense calcified plaques leads to an overestimation of lumen narrowing and false-positive results in stenosis rates (14). In this study, the fact that the majority of the patients had mixed and calcified plaques may have caused similar results.

While coronary artery plaques are visualized as regular or irregular filling defects in ICA images, MDCT coronary angiography allows for the characterization of plaques that cause luminal narrowing, thus clinically indicate more risky lesions. In this study, of the plaques identified by MDCT angiography, $48.3 \%(n=117)$ were mixed, $39.2 \%$ $(n=95)$ were calcified, and $12.3 \%(n=30)$ were soft. Studies have shown that soft-mixed plaques with surface irregularities or ulcerations are much more likely to cause acute coronary syndromes than calcified plaques (18).

\section{CONCLUSION}

In conclusion, this study revealed that MDCT coronary angiography provided high sensitivity and negative predictive values in the examination of segment-based and patient-based analyses. MDCT coronary angiography can be used as a suitable non-invasive diagnostic alternative to ICA in patients with known CAD or those included in the low or moderate risk groups. Congresses

\section{The study was represented as an e-poster at European Congress of Radiology in 2018.}

Financial disclosures: All authors report no financial 
interests or potential conflicts of interest.

Conflict of Interest: The authors declare that they have no competing interest.

Ethical approval: The study was approved by the Inonu University Medical Faculty Ethical Committee. (2015/16). All procedures performed in studies involving human participants were in accordance with the ethical standards of the institutional research committee and with the 1964 Helsinki declaration and its later amendments or comparable ethical standards.

\section{REFERENCES}

1. Benjamin, E. J., Muntner, P., Alonso, A., et al. Heart disease and stroke statistics 2019 update: a report from the American Heart Association. Circulation. 2019;39:e56-e528.

2. Murray CJ, Lopez AD. Mortality by cause for eight regions of the world: GlobalBurden of Disease Study. Lancet. 1997; 349: 1269-76.

3. American Heart Association 2002 Heart and Stroke Statistical Update. Dallas: Am Heart Association. 2001.

4. Kantarcı M, Duran C, Durur I, et al. Detection of myocardial bridging with ECG-gated MDCT and multiplanar reconstruction. AJR Am J Roentgenol. 2006;186.6 supplement_2:391-4.

5. Taylor AJ, Cerqueira M, Hodgson JM, et al. ACCF/ SCCT/ ACR/ AHA/ ASE/ASNC/ NASCl/ SCAI/ SCMR 2010 appropriate use criteria for cardiac computedtomography. J Am Coll Cardiol. 2010; 56: 1864-94.

6. Goerne, H., de la Fuente, D., Cabrera, M., et al. Imaging Features of Complications after Coronary Interventions and Surgical Procedures. RadioGraphics. 2021; 41(3), 699-719.

7. Seto, A. H., Shroff, A., Abu Fadel, et al. Length of stay following percutaneous coronary intervention: an expert consensus document update from the society for cardiovascular angiography and interventions. Catheterization and Cardiovascular Interventions. 2018;92:717-31.

8. Ohnesorge B, Flohr T, Becker C, et al. Cardiacimaging by means of electrocardiographically gated multisection spiral CT: initialexperience. Radiology. 2000;217:564-71.

9. Giesler T, Baum U, Ropers D, et al. Noninvasive visuallization of coronary arteriesusing contrast-enhanced multidedector CT: influence of heart rate on image quality andstenosis dedection. AJR Am J Roentgenol. 2002;179:911-16.
10. Abdulla J, Abildstrom SZ, Gotzsche O, et al. 64-multislice detector computed tomography coronary angiography as potential alternative to conventional coronary angiography: a systematic review and meta-analysis. Eur Heart $\mathrm{J}$. 2007;28:3042-50.

11. Hamon, M., Biondi-Zoccai, G. G., Malagutti,P., et al. Diagnostic performance of multislice spiral computed tomography of coronary arteries as compared with conventional invasive coronary angiography: a meta-analysis. J Am College Cardiol. 2006;48:1896-910.

12. Ehara M, Surmely JF, Kawai M et al. Diagnostic accuracy of 64-slice computedtomography for detecting angiographically significant coronary artery stenosis in anunselected consecutive patient population: comparison with conventional invasiveangiography. Circ J. 2006; 70:56471.

13. Scheffel H, Leschka S, Plass A et al. Accuracy of 64-slice computed tomographyfor the preoperative detection of coronary artery disease in patients with chronic aorticregurgitation. Am J Cardiol. 2007;100:701-6.

14. Chao SP, Law WY, Kuo CJ, et al. Diagnostic accuracy of 256-row computed tomographic angiography with invasive coronary angiography in patients with suspected coronary artery disease. Eur Heart J. 2010;31:1916-23.

15. Miller JM, Rochitte $C E$, Dewey $M$, et al. Diagnostic performance of coronaryangiography by 64 -row CT. N Engl J Med. 2008;359:2324-36.

16. Leber AW, Knez A, von Ziegler $F$, et al. Quantification of obstructive and nonobstructive coronary lesions by 64-slice computed tomography: a comparative study with quantitative coronary angiography and intravascular ultrasound. J Am Coll Cardiol. 2005;46:147-54.

17. Budoff MJ, Dowe D, Jollis JG, et al. Diagnostic performance of 64-multidetector row coronary computed tomographic angiography for evaluation of coronary artery stenosis in individuals without known coronary artery disease: results from the prospective multicenter ACCURACY (Assessment by Coronary Computed Tomographic Angiography of Individuals Undergoing Invasive Coronary Angiography) trial. J Am College of Cardiol. 2008; 52:1724-32.

18. Lutgens E, van Suylen RJ, Faber BC, et al. Atherosclerotic plaque rupture: local orsystemic process? Arterioscler Thromb Vasc Biol. 2003;23:2123-30. 